\title{
Spectral Efficiency of Dynamic Coordinated Beamforming: A Stochastic Geometry Approach
}

\author{
Namyoon Lee, David Morales-Jimenez, Angel Lozano, and Robert W. Heath Jr.
}

\begin{abstract}
This paper characterizes the performance of coordinated beamforming with dynamic clustering. A downlink model based on stochastic geometry is put forth to analyze the performance of such base station (BS) coordination strategy. Analytical expressions for the complementary cumulative distribution function (CCDF) of the instantaneous signal-to-interference ratio (SIR) are derived in terms of relevant system parameters, chiefly the number of BSs constituing the coordination clusters, the number of antennas, and the pathloss exponent. Utilizing this CCDF, with pilot overheads further incorporated into the analysis, we formulate the optimization of the BS coordination clusters for a given fading coherence. Our results indicate that (i) coordinated beamforming is most beneficial to users that are in the outer part of their cells yet in the inner part of their coordination cluster, and that (ii) the optimal cluster cardinality for the typical user is small and it scales with the fading coherence. Simulation results verify the exactness of the SIR distributions derived for stochastic geometries, which are further compared with the corresponding distributions for deterministic grid networks.
\end{abstract}

\section{INTRODUCTION}

\section{A. Background}

Base station (BS) coordination is regarded as an effective approach to mitigate intercell interference [2][4]. The idea is to allow multiple BSs to coordinate their transmit/receive strategies (e.g., beamforming, power control, and scheduling) by utilizing channel state information (CSI). The performance would increase monotonically with the number of coordinated BSs if such CSI could be acquired at no cost and thus, ideally, entire systems should be coordinated [5]-[9]. In practice though, coordination of an entire (large) network is not

N. Lee, and R. W. Heath Jr. are with the Wireless Networking and Communications Group, Department of Electrical and Computer Engineering, The University of Texas at Austin, Austin, TX 78712, USA. (e-mail:\{namyoon.lee, rheath\}@utexas.edu)

D. Morales-Jimenez and A. Lozano are with the Department of Information and Communication Technologies, Universitat Pompeu Fabra, 08018 Barcelona, Spain. (email: $\{$ d.morales,angel.lozano\}@upf.edu)

Parts of this paper are to be presented at the International Conference in Communications (ICC'14) [1] only computationally unfeasible, but undesirable once the ensuing overheads are taken into account [10]. A central concept in the implementation of BS coordination is then that of a cluster, defined as the set of BSs that a given user coordinates with. From the vantage of a user then, only those BSs outside the cluster are sources of interference. Intuitively, a larger cluster reduces intercell interference but it also increases the overheads required to acquire the necessary CSI at the BSs. It follows that determining the optimal cluster cardinality is a key step to assess the true benefits of BS coordination. This paper tackles such optimization for a particular coordination strategy.

\section{B. Related Work}

In toy setups where all the BSs can participate in the coordination, centralized schemes have been shown to yield sum spectral efficiencies that increase unboundedly with the transmit powers [5]-[8]. In large networks, where the CSI-acquisition overheads and the channel uncertainty caused by fading selectivity prevent large cluster cardinalities, out-of-cluster interference is inevitable and the spectral efficiency has been shown to be fundamentally bounded no matter how sophisticated the cooperation [9]-[13]. Nevertheless, small cooperation clusters not incurring too much overhead do provide performance improvements with respect to a noncooperative baseline. Since, despite their regularity, deterministic grid models are remarkably unfriendly to analysis, most of the results for grid networks are simulation-based. Analytical results are available only for the simplest embodiments thereof, in particular for the so-called Wyner model where BSs and mobile users are located along a one-dimensional universe [13], [14].

Approaches based on stochastic geometry are rapidly gaining momentum because of their superior analytical tractability and because they happen to match well the heterogeneous nature of emerging networks [15]. Within this framework, the performance of BS coordination schemes with fixed cluster structures established a-priori has been studied [16]-[18], [21].

Dynamic BS clustering is a way of forming cooperative BS sets such that users in a network select their own 
cooperation BSs based on users' locations and channel quality. Given the evidence (e.g., [19], [20], [23]) that dynamic clusters based on user locations and channel propagation features perform far better than their fixed counterparts (i.e., static BS clustering), there is clear interest in extending the existing stochastic geometry analyses to such dynamic cooperation structures.

\section{Contribution}

We consider the downlink of a network whose topology is modeled through stochastic geometry. BS locations are modeled as a homogeneous Poisson point process (PPP) with the corresponding cells determined from a tessellation of the plane into Voroni regions. Users in each cell are randomly located and each one then defines its own cluster, i.e., the set of BSs it coordinates with, on the basis of such location. Under this dynamic BS clustering policy, the set of users that share the same BS cluster are served through coordinated beamforming, a method that mitigates intra-cluster interference. Our contribution is to characterize the performance of such dynamic coordinated beamforming.

We derive analytical expressions for the signal-tointerference ratio (SIR) distributions and the ergodic spectral efficiency in terms of system parameters, chiefly the path-loss exponent, the number of antennas per BS, the cluster cardinality, and the pilot overhead. We obtain these analytical results for users with specific in-cluster relative locations and, by marginalizing over such locations, for the typical user. Utilizing this latter result, we then characterize the benefits of coordination in terms of the net ergodic spectral efficiency, incorporating the pilot overheads required for coordinated beamforming. From this, we obtain the optimal cluster cardinality for the typical user. Our finding is that coordinated beamforming is most beneficial to users that are in the outer part of their cells yet in the inner part of their coordination cluster, and that the optimal cluster cardinality for the typical user is small and scales with the fading coherence Through simulation, the accuracy of the derived SIR distributions is verified.

The remainder of the paper is organized as follows. Section II describes the proposed models as well as the performance metrics for the considered coordinated beamforming scheme. In Section III, analytical expressions for the SIR distribution are derived for specific relative in-cluster locations. The SIR distribution for the typical user is derived in Section IV, and then utilized in Section V to analyze the optimal cluster cardinality. Section VI concludes the paper.

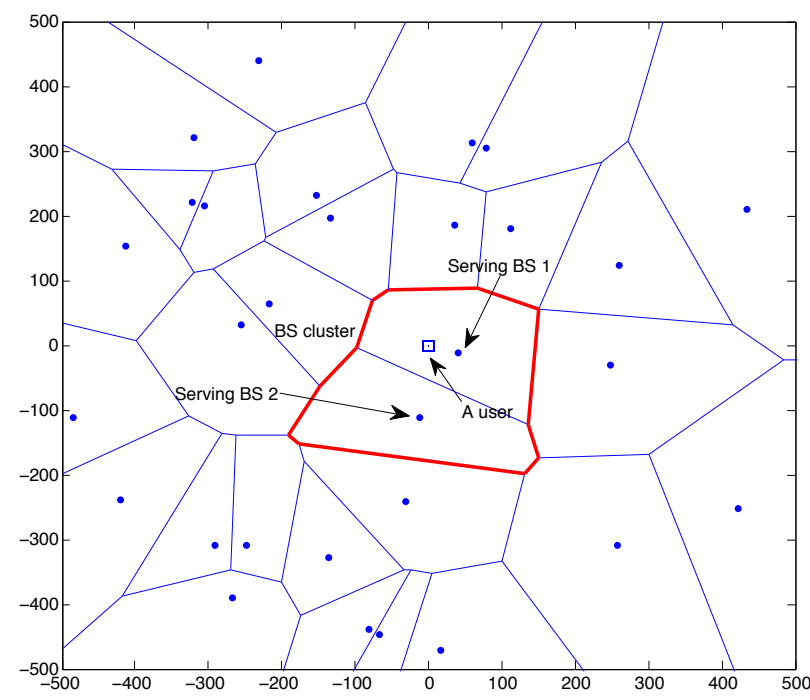

Fig. 1. A snapshot of the proposed model for $K=2$. A user connects with its two nearest BSs while receiving interference from the rest. The relative location of the first BS is determined by the parameter $\delta_{1}$ in our model.

\section{MOdELS AND METRICS}

\section{A. Network Model}

We consider a cellular network consisting of BSs, each equipped with $N_{\mathrm{t}}$ antennas. The locations of these BS are established according to a homogeneous PPP, $\Phi=\left\{d_{k}, k \in \mathbb{N}\right\}$, on the plane $\mathbb{R}^{2}$. This PPP has density $\lambda$. By tessellating the plane into Voronoi regions around each BS, we can define cells in the traditional manner. We further consider a population of singleantenna users distributed according to another homogeneous PPP, $\Phi_{\mathrm{U}}=\left\{u_{k}, k \in \mathbb{N}\right\}$, which has density $\lambda_{\mathrm{U}}$ and is independent of $\Phi$.

\section{B. Dynamic Clustering Model}

Without loss of generality, we can order the BSs in increasing distance from the user. The dynamic clustering technique that we analyze relies on the policy of having each user coordinate with the $K$ closest BSs, where $K \leq N_{\mathrm{t}}$ is the cardinality of the coordination clusters (cf. Fig. 1). For later use, we define $\delta_{1}=\left\|d_{1}\right\| /\left\|d_{K}\right\|$ as the distance to the closest BS normalized by that to the furthest in the cluster. Given that the user density is much higher than the BS density, i.e., $\lambda_{\mathrm{U}} \gg \lambda$, with high probability there shall be $K$ users that choose to connect with the same set of $K$ BSs. (For scenarios of lower user densities, $\lambda_{\mathrm{U}} \approx \lambda$, it is possible to consider user-centric coordination strategies involving the $K<N_{\mathrm{t}}$ nearest BSs.) 


\section{Signal Model}

Under the premise of separate encoding at each BS, the $k$ th BS sends an information symbol $s_{k}$ (intended for the $k$ th user) through a linear beamforming vector $\mathbf{v}_{k}=\left[v_{k}^{1}, v_{k}^{2}, \ldots, v_{k}^{N_{\mathrm{t}}}\right]^{T}$ with unit norm, $\left\|\mathbf{v}_{k}\right\|_{2}=1, k \in$ $\{1,2, \ldots, K\}$. Without loss of generality, let us focus on a user located at the origin. The observation at this user is

$$
\begin{aligned}
y_{1} & =\left\|d_{1}\right\|^{-\beta / 2} \mathbf{h}_{1,1} \mathbf{v}_{1} s_{1}+\sum_{k=2}^{K}\left\|d_{k}\right\|^{-\beta / 2} \mathbf{h}_{1, k} \mathbf{v}_{k} s_{k} \\
& +\sum_{k=K+1}^{\infty}\left\|d_{k}\right\|^{-\beta / 2} \mathbf{h}_{1, k} \mathbf{v}_{k} s_{k}+z_{1}
\end{aligned}
$$

where $\mathbf{h}_{1, k}=\left[h_{1, k}^{1}, h_{1, k}^{2}, \ldots, h_{1, k}^{N_{\mathrm{t}}}\right] \in \mathbb{C}^{1 \times N_{\mathrm{t}}}$ represents the downlink channel between the $k$ th $\mathrm{BS}$ and the user, with entries that are independent and identically distributed (IID) complex Gaussian random variables having zero mean and unit variance, i.e., $\mathcal{C N}(0,1)$. The channels vary over time in an IID block-faded fashion. Further, $\beta$ represents the pathloss exponent and $z_{1}$ denotes the additive Gaussian noise, $z_{1} \sim \mathcal{C N}\left(0, \sigma^{2}\right)$. The transmit power at each BS satisfies $\mathbb{E}\left[\left|s_{k}\right|^{2}\right] \leq P$.

Each user learns the downlink channels from the $K$ BSs within its cluster by means of orthogonal pilot symbols and then conveys this information back to the BSs via error-free feedback links. From this CSI, the coordinated beamforming scheme constructs the beamforming vectors $\mathbf{v}_{k}, k \in\{1, \ldots, K\}$, that nullify intracluster interference while maximizing the desired signal strength for the $K$ users in the cluster. Thus, the first BS selects beamforming vector $\mathbf{v}_{1}$ solving

$$
\begin{array}{ll}
\operatorname{maximize:} & \left|\mathbf{h}_{1,1} \mathbf{v}_{1}\right|^{2} \\
\text { subject to: } & \mathbf{h}_{k, 1} \mathbf{v}_{1}=0 \text { for } k \in\{2, \ldots, K\}, \\
& \left\|\mathbf{v}_{1}\right\|_{2}=1
\end{array}
$$

which always exists when $N_{\mathrm{t}} \geq K$. The corresponding instantaneous SIR for the user at the origin is

$$
\operatorname{SIR}\left(K, N_{\mathrm{t}}, \beta\right)=\frac{\left|\mathbf{h}_{1,1} \mathbf{v}_{1}\right|^{2}\left\|d_{1}\right\|^{-\beta}}{I_{K}} .
$$

where

$$
I_{K}=\sum_{k=K+1}^{\infty}\left|\mathbf{h}_{1, k} \mathbf{v}_{k}\right|^{2}\left\|d_{k}\right\|^{-\beta}
$$

is the aggregate out-of-cluster interference power. This instantaneous SIR in (3) involves multiple levels of randomness:

1) The randomness associated with the user location relative to its serving $\mathrm{BS}$; this is incorporated through $\left\|d_{1}\right\|$. Equivalently, and more conveniently to the analysis that follows later, it can be incorporated through $\left\|d_{K}\right\|$ and $\delta_{1}=\frac{\left\|d_{1}\right\|}{\left\|d_{K}\right\|}$.

2) The randomness associated with the user location relative to the interfering BSs; this is incorporated through $\left\|d_{k}\right\|, k>K$.

3) The randomness associated with the desired link fading; this is incorporated through $\mathbf{h}_{1,1}$.

4) The randomness associated with the interference fading; this is incorporated through $\mathbf{h}_{1, k}, k>K$.

\section{Performance Metrics}

The CCDF of the instantaneous SIR is characterized at two different levels, with the absolute dimensions of the network abstracted out.

1) Specific Relative Cluster Geometry: First, we characterize the CCDF of the SIR for some given relative distances $\left\{\delta_{1}, \ldots, \delta_{K}\right\}$, but with the absolute distances $\left\{d_{1}, \ldots, d_{K}\right\}$ and the out-of-cluster interference $I_{K}$ marginalized over. Since the signals received from BSs $k=2, \ldots, K$ do not contribute interference by virtue of (2), it suffices to condition on $\delta_{1}$ and the ensuing conditional CCDF is

$$
\begin{aligned}
& F_{\mathrm{SIR} \mid \delta_{1}}^{\mathrm{c}}\left(K, N_{\mathrm{t}}, \beta, \delta_{1} ; \gamma\right)=\mathbb{P}\left[\operatorname{SIR}\left(K, N_{\mathrm{t}}, \beta\right) \geq \gamma \mid \delta_{1}\right] \\
& =\mathbb{E}\left[\mathbb{P}\left[\frac{\left|\mathbf{h}_{1,1} \mathbf{v}_{1}\right|^{2}\left(\delta_{1}\left\|d_{K}\right\|\right)^{-\beta}}{I_{K}} \geq \gamma \mid \delta_{1},\left\|d_{K}\right\|, I_{K}\right] \mid \delta_{1}\right]
\end{aligned}
$$

where the expectation over $\left\|d_{K}\right\|$ and $I_{K}$, characterized in Section III, effect the marginalization. This conditional CCDF does not correspond to the distribution of the SIR experience by any actual user in the system, but it is representative of the average behavior in all possible cluster geometries that share a particular $\delta_{1}$, i.e., a particular relative location of the user within the cluster. This SIR distribution should be interpreted carefully because $\delta_{1}$ is dependent of the cluster cardinality $K$. Intuitively, $\delta_{1} \ll 1$ represents geometries where the user is located near the cluster center whereas $\delta_{1} \approx 1$ indicates that the user is near the cluster edge.

2) Average Cluster Geometry: By further marginalizing over $\delta_{1}$, we obtain the SIR distribution averaged over all possible geometries, which is less informative than the one in (6). In particular, this fully marginalized distribution does not allow discriminating between situations that are either favorable or adverse to coordinated beamforming, but it does serve as a stepping stone towards the computation of average quantities. The fully 
marginalized CCDF of the SIR is

$$
\begin{aligned}
& F_{\mathrm{SIR}}^{\mathrm{c}}\left(K, N_{\mathrm{t}}, \beta ; \gamma\right)=\mathbb{P}\left[\operatorname{SIR}\left(K, N_{\mathrm{t}}, \beta\right) \geq \gamma\right] \\
& =\mathbb{E}\left[\mathbb{P}\left[\frac{\left|\mathbf{h}_{1,1} \mathbf{v}_{1}\right|^{2}\left(\delta_{1}\left\|d_{K}\right\|\right)^{-\beta}}{I_{K}} \geq \gamma \mid \delta_{1},\left\|d_{K}\right\|, I_{K}\right]\right] .
\end{aligned}
$$

where the expectation is now also over $\delta_{1}$ in addition to $\left\|d_{K}\right\|$ and $I_{K}$. This distribution will be used to characterize the performance of the typical user in Secs. IV and V.

\section{Specific Relative Cluster Geometry}

In this section, we characterize the conditional CCDF in (6) in terms of $K, N_{\mathrm{t}}$ and $\beta$.

\section{A. General Characterization}

We begin by providing a general characterization in integral form.

Theorem 1. For a given $\delta_{1}$,

$$
\begin{aligned}
& F_{\mathrm{SIR} \mid \delta_{1}}^{\mathrm{c}}\left(K, N_{\mathrm{t}}, \beta, \delta_{1} ; \gamma\right) \\
& =\mathbb{E}\left[\left.\sum_{m=0}^{N_{\mathrm{t}}-K} \frac{r^{\beta m}}{m !}(-1)^{m} \frac{\mathrm{d}^{m} \mathcal{L}_{\tilde{I}_{r}}(s)}{\mathrm{d} s^{m}}\right|_{s=r^{\beta}}\right]
\end{aligned}
$$

where $\tilde{I}_{r}=\delta_{1}^{\beta} \gamma \sum_{k=K+1}^{\infty} H_{k}\left\|d_{k}\right\|^{-\beta}$ while $\mathcal{L}_{\tilde{I}_{r}}(s)=$ $\mathbb{E}\left[e^{-s \tilde{I}_{r}}\right]$ denotes the Laplace transform of $\tilde{I}_{r}$, which is given in (53) in Appendix B, and the expectation is over $\left\|d_{K}\right\|=r$, distributed as per Lemma 3 .

Proof. See Appendix A.

Although general and exact, the form given in Theorem 1 is rather unwieldy, motivating the interest in more compact characterizations. Still in full generality, we next provide closed-form upper and lower bounds to the distribution.

Theorem 2. For a given $\delta_{1}$,

$$
\begin{aligned}
& F_{\mathrm{SIR} \mid \delta_{1}}^{\mathrm{c}, \mathrm{L}}\left(K, N_{\mathrm{t}}, \beta, \delta_{1} ; \gamma\right) \leq F_{\mathrm{SIR} \mid \delta_{1}}^{\mathrm{c}}\left(K, N_{\mathrm{t}}, \beta, \delta_{1} ; \gamma\right) \\
& F_{\mathrm{SIR} \mid \delta_{1}}^{\mathrm{c}}\left(K, N_{\mathrm{t}}, \beta, \delta_{1} ; \gamma\right) \leq F_{\mathrm{SIR} \mid \delta_{1}}^{\mathrm{c}, \mathrm{U}}\left(K, N_{\mathrm{t}}, \beta, \delta_{1} ; \gamma\right)
\end{aligned}
$$

with

$$
\begin{aligned}
& F_{\mathrm{SIR} \mid \delta_{1}}^{\mathrm{c}, \mathrm{U}}\left(K, N_{\mathrm{t}}, \beta, \delta_{1} ; \gamma\right)=\sum_{\ell=1}^{N_{t}-K+1} \frac{\left(\begin{array}{c}
N_{t}-K+1 \\
\ell
\end{array}\right)(-1)^{\ell+1}}{\left[1+\mathcal{D}\left(\ell \kappa \delta_{1}^{\beta} \gamma, \beta\right)\right]^{K}} \\
& F_{\mathrm{SIR} \mid \delta_{1}}^{\mathrm{c}, \mathrm{L}}\left(K, N_{\mathrm{t}}, \beta, \delta_{1} ; \gamma\right)=\sum_{\ell=1}^{N_{t}-K+1} \frac{\left(\begin{array}{c}
N_{t}-K+1 \\
\ell
\end{array}\right)(-1)^{\ell+1}}{\left[1+\mathcal{D}\left(\ell \delta_{1}^{\beta} \gamma, \beta\right)\right]^{K}}
\end{aligned}
$$

where $\kappa=\left(N_{t}-K+1\right) !^{\frac{-1}{N_{t}-K+1}}$ and

$$
\mathcal{D}(A, B)=\frac{2 A}{B-2}{ }_{2} F_{1}\left(1,1-\frac{2}{B}, 2-\frac{2}{B},-A\right)
$$

with ${ }_{2} F_{1}(\cdot)$ the Gauss hypergeometric function.

\section{Proof. See Appendix B.}

The upper and lower bounds coincide when $N_{\mathrm{t}}=K$, implying that for this most important case we obtain the exact CCDF.

Corollary 1. For a given $\delta_{1}$ and $K=N_{\mathrm{t}}$,

$$
F_{S I R \mid \delta_{1}}^{\mathrm{c}}\left(K, K, \beta, \delta_{1} ; \gamma\right)=\frac{1}{\left[1+\mathcal{D}\left(\delta_{1}^{\beta} \gamma, \beta\right)\right]^{K}} .
$$

\section{B. Special Cases}

To shed further light on the significance of the expressions in Thm. 2 and Cor. 1, it is instructive to consider certain special cases.

1) Noncoordinated Network: The most basic special case is the one where there is no coordinated beamforming, i.e., where $K=1$ (and $\delta_{1}=1$ with the conditioning thereupon immaterial). By setting $N_{\mathrm{t}}=1$ we then recover the CCDF of the SIR given in [15], namely

$$
F_{\mathrm{SIR}}^{\mathrm{c}}(1,1, \beta ; \gamma)=\frac{1}{1+\mathcal{D}(\gamma, \beta)}
$$

which Thm. 2 therefore generalizes. For this special case, the derived expressions are useful to characterize the benefits of having $N_{\mathrm{t}}$ antennas. As illustrated in Fig. 2, the upper bound tightly matches the exact CCDF over the entire range of SIRs of interest and for distinct values of $N_{\mathrm{t}}$, i.e., $F_{\mathrm{SIR}}^{\mathrm{c}, \mathrm{U}}\left(1, N_{\mathrm{t}}, \beta, 1 ; \gamma\right) \simeq F_{\mathrm{SIR}}^{\mathrm{c}}\left(1, N_{\mathrm{t}}, \beta, 1 ; \gamma\right)$.

2) Coordinated Network with $N_{\mathrm{t}}=K$ : With the coordinated beamforming activated and $N_{\mathrm{t}}=K$, the behavior is characterized by the simple expression in Cor. 1. Particularized to $\beta=4$, which is a standard value in terrestrial outdoor wireless systems, $\mathcal{D}(\cdot, \cdot)$ reduces to

$$
\mathcal{D}(\xi, 4)=\sqrt{\xi} \operatorname{arccot}\left(\frac{1}{\sqrt{\xi}}\right)
$$

which, plugged into (15), yields

$$
F_{\mathrm{SIR} \mid \delta_{1}}^{\mathrm{c}}\left(K, K, 4, \delta_{1} ; \gamma\right)=\frac{1}{\left[1+\sqrt{\gamma} \delta_{1}^{2} \operatorname{arccot}\left(\frac{1}{\sqrt{\gamma} \delta_{1}^{2}}\right)\right]^{K}}
$$

This simple CCDF facilitates gauging different scenarios as indicated earlier: $\delta_{1} \ll 1$ corresponds to users located in the central part of the cluster while $\delta_{1} \approx 1$ corresponds to users located near the cluster edge. Specifically, as 


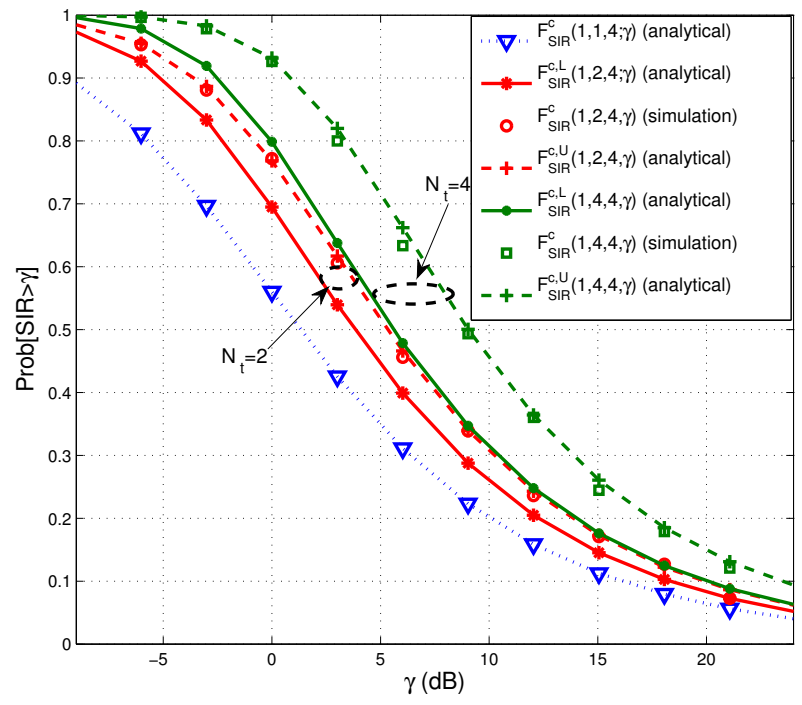

Fig. 2. CCDF of the SIR for $\beta=4$ and different numbers of transmit antennas in the absence of coordinated beamforming.

TABLE I

ERGODIC SPECTRAL EFFICIENCY (BITS/S/Hz) OF COORDINATED BEAMFORMING FOR $N_{\mathrm{t}}=K=2, C\left(2,2,4, \delta_{1}\right)$

\begin{tabular}{c|c|c|c}
\hline Relative location $\delta_{1}$ & $\delta_{1}=\frac{1}{3}$ & $\delta_{1}=\frac{1}{2}$ & $\delta_{1}=\frac{2}{3}$ \\
\hline \hline$C\left(2,2,4, \delta_{1}\right)$ & 5.377 & 3.3361 & 2.1318 \\
\hline
\end{tabular}

illustrated in Fig 3, when $K=N_{t}=2$ and $\beta=4$, a user with $\delta_{1}=0.5$ (in red) has a better SIR distribution (and thus higher benefits from coordinated beamforming) than a user with $\delta_{1}=0.8$ (in blue). A similar observation can be made for $\delta_{1}=1 / 4$ and $\delta_{1}=1 / 3$ when $K=N_{t}=$ $\beta=4$.

The following example further illustrates benefits of coordinated beamforming in terms of ergodic spectral efficiency for different relative locations, $\delta_{1}$.

Example 1: For $N_{\mathrm{t}}=K=2$ and $\beta=4$, the ergodic spectral efficiency (in bits/s/Hz) is

$$
\begin{gathered}
C\left(2,2,4, \delta_{1}\right)=\int_{0}^{\infty} \log _{2}(1+\gamma) \mathrm{d} F_{\mathrm{SIR} \mid \delta_{1}}\left(2,2,4, \delta_{1}\right) \\
=\int_{0}^{\infty} \frac{\log _{2} e}{(1+\gamma)} F_{\mathrm{SIR} \mid \delta_{1}}^{\mathrm{c}}\left(2,2,4, \delta_{1}\right) \\
=\int_{0}^{\infty} \frac{\log _{2} e}{(1+\gamma)\left[1+\sqrt{\gamma} \delta_{1}^{2} \operatorname{arccot}\left(1 / \sqrt{\gamma} \delta_{1}^{2}\right)\right]^{2}} \mathrm{~d} \gamma
\end{gathered}
$$

where in (20) integration by parts was applied. The values for different relative locations $\delta_{1}$, listed in Table I, reveal that the most substantial gains of coordinated beamforming are obtained for small $\delta_{1}$, which corresponds to cluster-center areas. This is because clusteredge users remain subject to strong interference from out-of-cluster BSs.

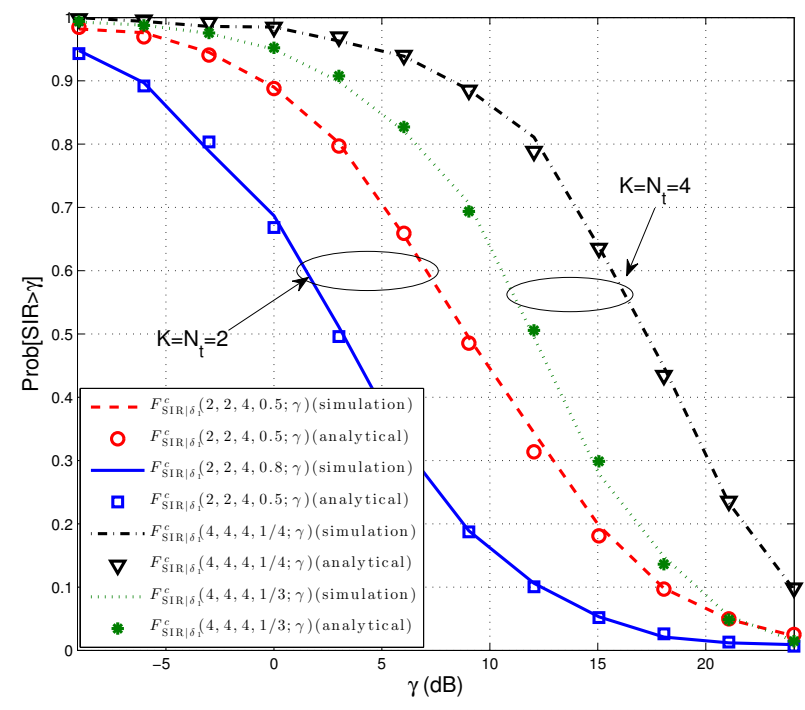

Fig. 3. Conditional CCDF of the SIR for $\beta=4$ and $K=N_{\mathrm{t}} \in$ $\{2,4\}$.

\section{Average Cluster Geometry}

This section is devoted to the characterization of the CCDF in (8). Specifically, tight lower and upper bounds are derived as a function of $K, N_{\mathrm{t}}$ and $\beta$.

\section{A. Upper and Lower Bounds}

The following Lemma provides the probability density function (PDF) of $\delta_{1}=\frac{\left\|d_{1}\right\|}{\left\|d_{K}\right\|}$ induced by the underlying PPP.

Lemma 1 (PPP distance ratio). Let $\left\|d_{1}\right\|$ and $\left\|d_{K}\right\|$ denote the distances from the origin to the first and the Kth BSs. The PDF of $\delta_{1}=\frac{\left\|d_{1}\right\|}{\left\|d_{K}\right\|}$ is given by

$$
f_{\delta_{1}}(x)=2(K-1) x\left(1-x^{2}\right)^{K-2} \quad \text { for } \quad 0 \leq x \leq 1 .
$$

\section{Proof. See Appendix C.}

Applying the above lemma to (12) and (13) we can readily write

$$
\begin{aligned}
& F_{\text {SIR }}^{\mathrm{c}, \mathrm{U}}\left(K, N_{\mathrm{t}}, \beta ; \gamma\right) \\
& =\sum_{\ell=1}^{N_{\mathrm{t}}-K+1}\left(\begin{array}{c}
N_{\mathrm{t}}-K+1 \\
\ell
\end{array}\right)(-1)^{\ell+1} \int_{0}^{1} \frac{f_{\delta_{1}}(x)}{\left[1+\mathcal{D}\left(\ell \kappa x^{\beta} \gamma, \beta\right)\right]^{K}} \mathrm{~d} x \\
& F_{\text {SIR }}^{\mathrm{c}, \mathrm{L}}\left(K, N_{\mathrm{t}}, \beta ; \gamma\right) \\
& =\sum_{\ell=1}^{N_{\mathrm{t}}-K+1}\left(\begin{array}{c}
N_{\mathrm{t}}-K+1 \\
\ell
\end{array}\right)(-1)^{\ell+1} \int_{0}^{1} \frac{f_{\delta_{1}}(x)}{\left[1+\mathcal{D}\left(\ell x^{\beta} \gamma, \beta\right)\right]^{K}} \mathrm{~d} x
\end{aligned}
$$

for which analytical approximations are derived next. 


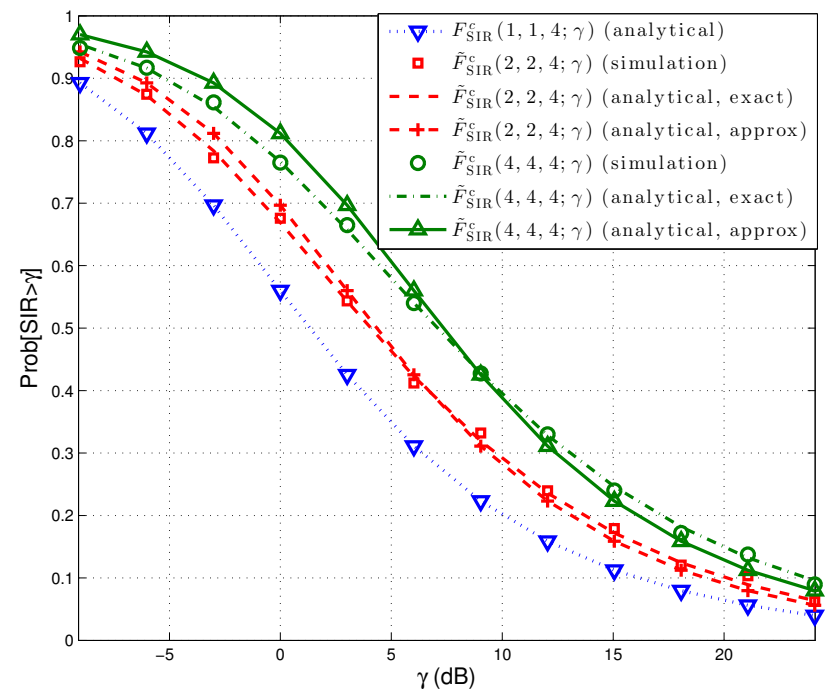

Fig. 4. CCDF of the SIR for different cluster cardinalities with $\beta=4$ and $N_{\mathrm{t}}=K$.

\section{B. Bound Approximations}

As shown in App. D, the integrals within (23) and (24) satisfy

$$
\int_{0}^{1} \frac{2(K-1) u\left(1-u^{2}\right)^{K-2}}{\left[1+\mathcal{D}\left(\tilde{\gamma} u^{\beta}, \beta\right)\right]^{K}} \mathrm{~d} u \simeq \frac{1}{1+\frac{\tilde{\gamma}^{2 / \beta}}{\sqrt{K}} \mathcal{A}\left(\frac{\sqrt{K}}{\tilde{\gamma}^{2 / \beta}}\right)}
$$

where $\tilde{\gamma}=\kappa \ell \gamma$ and $\mathcal{A}(y)=\int_{y}^{\infty} \frac{1}{1+v^{\frac{\beta}{2}}} \mathrm{~d} v$. From (25), the bounds to the unconditioned CCDF of the SIR in turn satisfy

$$
\begin{aligned}
& F_{\mathrm{SIR}}^{\mathrm{c}, \mathrm{U}}\left(K, N_{\mathrm{t}}, \beta ; \gamma\right) \simeq \sum_{\ell=1}^{N_{\mathrm{t}}-K+1} \frac{\left(\begin{array}{c}
N_{\mathrm{t}}-K+1 \\
\ell
\end{array}\right)(-1)^{\ell+1}}{1+\frac{(\kappa \ell \gamma)^{2 / \beta}}{\sqrt{K}} \mathcal{A}\left(\frac{\sqrt{K}}{(\kappa \ell \gamma)^{2 / \beta}}\right)} \\
& F_{\mathrm{SIR}}^{\mathrm{c}, \mathrm{L}}\left(K, N_{\mathrm{t}}, \beta ; \gamma\right) \simeq \sum_{\ell=1}^{N_{\mathrm{t}}-K+1} \frac{\left(\begin{array}{c}
N_{\mathrm{t}}-K+1 \\
\ell
\end{array}\right)(-1)^{\ell+1}}{1+\frac{(\ell \gamma)^{2 / \beta}}{\sqrt{K}} \mathcal{A}\left(\frac{\sqrt{K}}{(\ell \gamma)^{2 / \beta}}\right)}
\end{aligned}
$$

which, for the most relevant case where $\beta=4$ and $N_{\mathrm{t}}=$ $K$, coincide yielding

$$
F_{\mathrm{SIR}}^{\mathrm{c}}(K, K, 4 ; \gamma) \simeq \frac{1}{1+\sqrt{\frac{\gamma}{K}} \operatorname{arccot}\left(\sqrt{\frac{K}{\gamma}}\right)} .
$$

The simple expression in (28) clearly shows how coordination with the $K$ nearest BSs improves the CCDF of the SIR with $K$ because $\operatorname{arccot}(x)$ is a decreasing function of $x$. Furthermore, this approximation recovers the exact CCDF of the SIR for $K=1$.

To validate (26) and (27), we compare them with simulation results in Fig. 4. The agreement is excellent for the various values of $K$ considered and the entire range of SIRs of interest. As $K$ increases, the SIR improves because the out-of-cluster interference abates, but that would come at the expense of further overheads. This motivates the optimization of $K$ with the overhead incorporated, a problem that is tackled in Section V.

\section{Low-SIR Analysis}

To wrap up our characterization of the SIR distribution under coordinated beamforming, we specialize it to the low-SIR regime, which is of particular relevance for the purposes of establishing communication outages in quasi-static communication settings [30].

Proposition 1. The unconditioned CCDF of the SIR for $K=N_{\mathrm{t}}$ expands as

$$
\begin{aligned}
F_{S I R}^{\mathrm{c}}(K, K, \beta ; \gamma) & =1-\frac{K(K-1)}{\beta-2} \frac{\Gamma\left(\frac{\beta}{2}+1\right) \Gamma(K-1)}{\Gamma\left(\frac{\beta}{2}+K\right)} \gamma \\
& +o(\gamma)
\end{aligned}
$$

Proof. See Appendix E.

For $\beta=4$, the expansion in (29) further simplifies into

$$
F_{\text {SIR }}^{\mathrm{c}}(K, K, \beta ; \gamma)=1-\frac{\gamma}{K+1}+o(\gamma)
$$

which evidences that, by allowing coordinated beamforming from the $K$ nearest BSs, the outage probability in a quasi-static setting would decrease linearly with $K$.

\section{Optimal Cluster Cardinality}

Having characterized the SIR distributions of a user at a specific relative location and of the typical user, in this section we establish the corresponding optimal (in terms of ergodic spectral efficiency) cluster cardinalities. To compute the effective ergodic spectral efficiency, we incorporate pilot overheads into the formulation so as to account for the cost of acquiring the CSI required for the coordinated beamforming.

Although we have ignored background noise in the analysis of SIR distributions, we take it into account for the purpose of pilot transmissions with out-of-cluster interference, as these take place in an orthogonal fashion among users within a cluster. For a given fading coherence $L_{\mathrm{b}}$ (in symbols), the pilot overhead is

$$
\alpha=\frac{L_{\mathrm{p}}\left(K, N_{\mathrm{t}}, \mathrm{SINR}\right)}{L_{\mathrm{b}}},
$$

where $L_{\mathrm{p}}\left(K, N_{\mathrm{t}}, \mathrm{SINR}\right)$ denotes the number of symbols reserved for pilots. This number varies with $K$ and $N_{\mathrm{t}}$, and also with the pilot-transmission SINR. 
We henceforth model the number of pilot symbols as $L_{\mathrm{p}}\left(K, N_{\mathrm{t}}, \mathrm{SINR}\right)=\eta K N_{\mathrm{t}}$ where $\eta \geq 1$ is an SINRdependent parameter that signifies the number of pilots per transmit antenna and fading coherence interval; the value of $\eta$ approaches 1 for SINR $\rightarrow \infty$ [29].

With the SIR distributions obtained in Secs. III and IV, we compute the optimal $K$ for different situations.

\section{A. Optimal Cluster Cardinality for a Specific Relative Cluster Geometry}

Assuming that perfect CSI is gathered from the pilot observations at the receiver and complex Gaussian codebooks are used, the ergodic spectral efficiency (in $\mathrm{bits} / \mathrm{s} / \mathrm{Hz}$ ) for a specific relative cluster geometry is

$$
\begin{aligned}
& C\left(K, N_{\mathrm{t}}, \beta, \delta_{1}, \alpha\right) \\
& =(1-\alpha) \int_{0}^{\infty} \log _{2}(1+\gamma) \mathrm{d} F_{\mathrm{SIR} \mid \delta_{1}}\left(K, N_{\mathrm{t}}, \beta, \delta_{1} ; \gamma\right) \\
& =(1-\alpha) \int_{0}^{\infty} \frac{F_{\mathrm{SIR} \mid \delta_{1}}^{\mathrm{c}}\left(K, N_{\mathrm{t}}, \beta, \delta_{1} ; \gamma\right)}{(1+\gamma) \log _{e} 2} \mathrm{~d} \gamma
\end{aligned}
$$

from which the cluster cardinality $K^{\star}$ that optimizes the effective spectral efficiency for a specific relative cluster geometry is obtained as the solution of the integer optimization

$$
K^{\star}\left(\delta_{1}\right)=\max _{K \in\left\{1,2, \ldots, N_{\mathrm{t}}\right\}} C\left(K, N_{\mathrm{t}}, \beta, \delta_{1}, \alpha\right)
$$

which can be obtained by means of a numerical line search technique. Notice that, under the relative cluster geometry characterization, $K^{\star}$ is solely a function of $\delta_{1}=\frac{\left\|d_{1}\right\|}{\left\|d_{K}\right\|}$. Thus, if the serving BS distance is fixed to $\left\|d_{1}\right\|, \delta_{1}$ decreases as $K$ (and thus $\left\|d_{K}\right\|$ ) increases.

Fig. 5 shows the effective ergodic spectral efficiency for $\delta_{1}=\left\{\sqrt{\frac{0.1}{K}}, \sqrt{\frac{0.3}{K}}, \sqrt{\frac{0.5}{K}}\right\}$ with parameters $L_{\mathrm{b}} / \eta=$ $200, \beta=4$, and $N_{\mathrm{t}}=K$. As shown in this figure, $K^{\star}$ increases with $\delta_{1}$ because cluster-edge users benefit the most from a cluster enlargement-even if their spectral efficiencies remain lower.

\section{B. Optimal Cluster Cardinality for an Average Cluster Geometry}

The effective spectral efficiency averaged over $\delta_{1}$ is $C\left(K, N_{\mathrm{t}}, \beta, \alpha\right)=(1-\alpha) \int_{0}^{\infty} \frac{\log _{2} e}{(1+\gamma)} F_{\mathrm{SIR}}^{\mathrm{c}}\left(K, N_{\mathrm{t}}, \beta ; \gamma\right) \mathrm{d} \gamma$

from which the optimal cluster cardinality $K^{\star}$ for an average cluster geometry is obtained as the solution of the integer optimization

$$
K^{\star}=\max _{K \in\left\{1,2, \ldots, N_{\mathrm{t}}\right\}} C\left(K, N_{\mathrm{t}}, \beta, \alpha\right)
$$

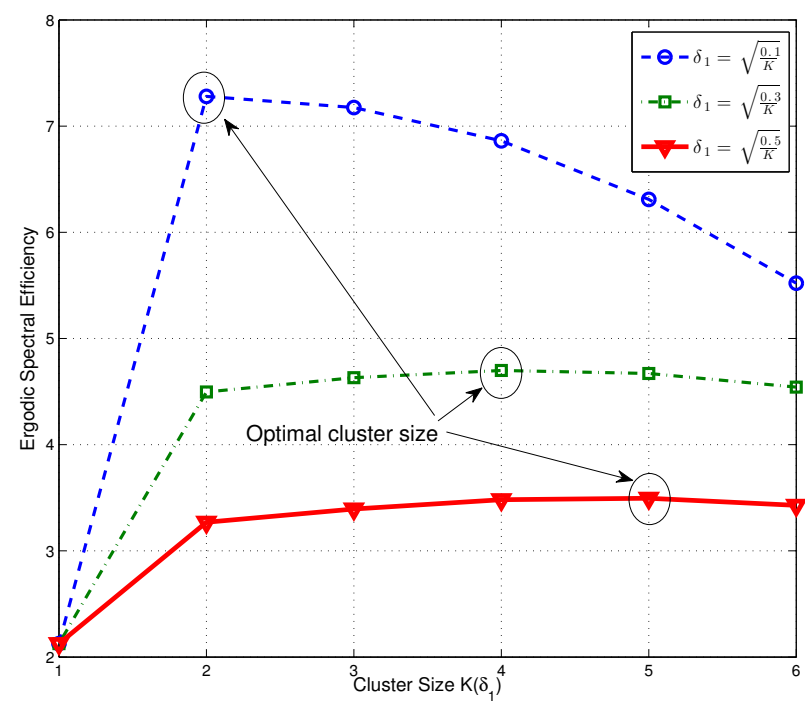

Fig. 5. Ergodic spectral efficiency as a function of $K$ with a fixed channel coherence parameter $\frac{L_{\mathrm{b}}}{\eta}=200$.

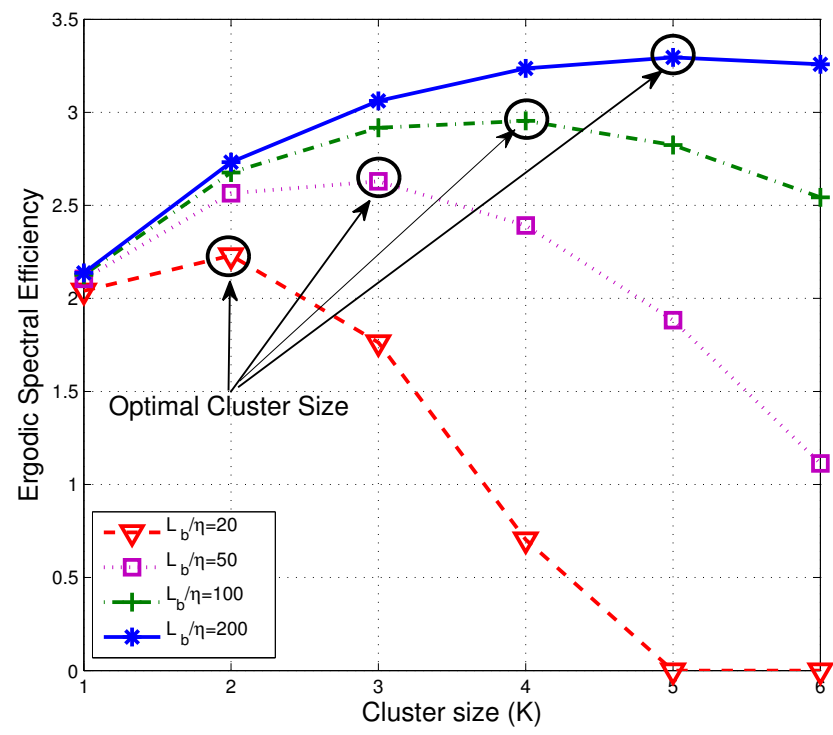

Fig. 6. Ergodic spectral efficiency as a function of $K$ for different channel coherence parameters, $\frac{L_{\mathrm{b}}}{\eta} \in\{20,50,100,200\}$.

whose solution can be obtained by a numerical line search technique.

Fig. 6 depicts the effective spectral efficiency as a function of $K$ for different ratios $L_{\mathrm{b}} / \eta$, with $\beta=4$ and $N_{\mathrm{t}}=K$. Notice how the optimum cluster cardinality increases with the channel coherence relative to the pilot cost, ranging from $K^{\star}=2$ when $L_{\mathrm{b}} / \eta=20$ to $K^{\star}=5$ when $L_{\mathrm{b}} / \eta=200$. For $N_{\mathrm{t}}=K=4$, the effective average spectral efficiencies for different fading coherences are summarized in Table II.

The optimal cluster cardinality with a short fading 
TABLE II

EfFective Average Spectral EfFiciency (BIts/s/Hz) of CoORdinated BeAmforming for $N_{\mathrm{t}}=4$

\begin{tabular}{c|c|c|c|c}
\hline Cluster Cardinality $K$ & 1 & 2 (Gain w.r.t. $K=1)$ & 3 (Gain w.r.t. $K=1)$ & 4 (Gain w.r.t. $K=1)$ \\
\hline \hline No pilot overhead $(\alpha=0)$ & 3.968 & $5.018(26.4 \%)$ & $4.249(7.1 \%)$ & $3.517(-11.4 \%)$ \\
\hline$L_{\mathrm{b}} / \eta=200\left(\alpha=\frac{K N_{\mathrm{t}}}{200}\right)$ & 3.889 & $4.817(23.8 \%)$ & $3.994(2.7 \%)$ & $3.236(-16.8 \%)$ \\
\hline$L_{\mathrm{b}} / \eta=20\left(\alpha=\frac{K N_{\mathrm{t}}}{20}\right)$ & 3.174 & $3.011(-5.2 \%)$ & $1.699(-46 \%)$ & $0.703(-78.1 \%)$ \\
\hline
\end{tabular}

coherence $\left(L_{\mathrm{b}} / \eta=20\right)$ is $K^{\star}=1$, implying that the cost of the coordination is in this case higher than the return. On the other hand, for a relatively long coherence $\left(L_{\mathrm{b}} / \eta=200\right)$ the optimal cluster cardinality is $K^{\star}=2$ with a $23.8 \%$ gain in average spectral efficiency relative to the $K=1$ baseline.

\section{COMPARISONS}

In this section, we compare the SIR distributions derived under our stochastic geometry model with the corresponding results-obtained through simulationfor a deterministic grid model. Since grid and PPP stochastic geometry models correspond to optimistic and pessimistic scenarios of real BS deployments as argued in [15], this comparison can be seen to convey upper and lower bounds to the actual benefits of dynamic coordinated beamforming.

In a deterministic square network, the BSs are arranged into a periodic square lattice on $\mathbb{R}^{2}$. For simulation purposes (cf. Fig. 7), we consider 36 BSs located at regular grid points and drop a user uniformly within the square highlighted in blue. Since we are considering dynamic clustering, in each realization the user selects its $K$ nearest BSs for coordination and the rest of the BSs constitute sources of interference. Without loss of generality, we can again index the BSs in increasing distance from the user and express the SIR as

$$
\mathrm{SIR}_{\text {grid }}=\frac{H_{1}\left\|d_{1}\right\|^{-\beta}}{\sum_{k=K+1}^{36} H_{k}\left\|d_{k}\right\|^{-\beta}}
$$

where $H_{k}$ and $\left\|d_{k}\right\|$ denote fading coefficient and distance from the $k$ th nearest BS to the user, respectively. In particular, $H_{1}$ has a Chi-squared distribution with $N_{\mathrm{t}}-K+1$ degrees of freedom. Meanwhile, the fading of all the interfering links, $\left\{H_{K+1}, H_{K+2}, \ldots, H_{36}\right\}$, are exponential with unit mean. Then, the CCDF of the SIR is

$F_{\text {SIR }_{\text {grid }}}^{\mathrm{c}}\left(K, N_{\mathrm{t}}, \beta ; \gamma\right)=\mathbb{E}\left[\mathbb{P}\left[\operatorname{SIR}_{\text {grid }}>\gamma \mid\left\|d_{1}\right\|,\left\{\left\|d_{k}\right\|, H_{k}\right\}\right]\right]$, where the expectation is over $\left\|d_{1}\right\|$ and $\left\{\left\|d_{k}\right\|, H_{k}\right\}$ for $k \in\{K+1, \ldots, 36\}$.

Fig. 8 shows the CCDFs for the two different models. As one would expect, the CCDF of the SIR in the deterministic grid model is somewhat more favorable than

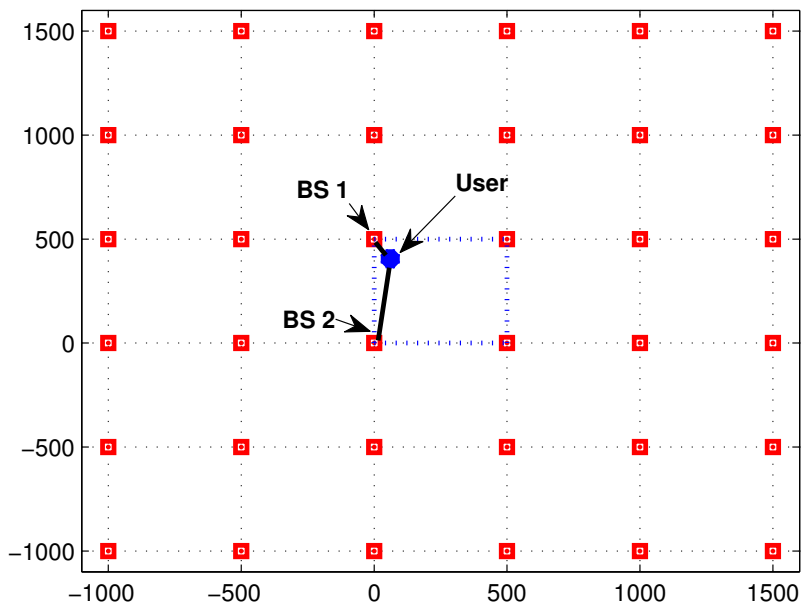

Fig. 7. A snapshot of the grid model for $K=2$. A user is uniformly located within the square region highlighted in blue, and it selects its two nearest BSs for coordination. In this snapshot, these BSs are the ones located at $(0,0)$ and $(0,500)$.

in the stochastic geometry model because, under PPP, the nearest interferer's location can be arbitrarily close to the in-cluster BSs. Nevertheless, the shapes of the CCDFs in the two models are analogous, which allows us to anticipate the gains by coordinated beamforming in actual deployments.

\section{CONCLUSION}

In this paper, we have characterized the performance of coordinated beamforming with dynamic BS clusters. Capitalizing on the tools of stochastic geometry, we have derived SIR distributions in terms of the number of BSs per cluster, the number of antennas per BS and the pathloss exponent. From these distributions, we have obtained analytical expressions for the effective ergodic spectral efficiency and optimized the cluster cardinality as function of the fading coherence. Our key finding is that coordinated beamforming is most beneficial to users in the inner part of the coordination clusters as opposed to users near the edges, where the mitigation of in-cluster interference makes less of a difference because of the strong out-of cluster interference component. Further, we have found that the optimal cluster cardinality for the 


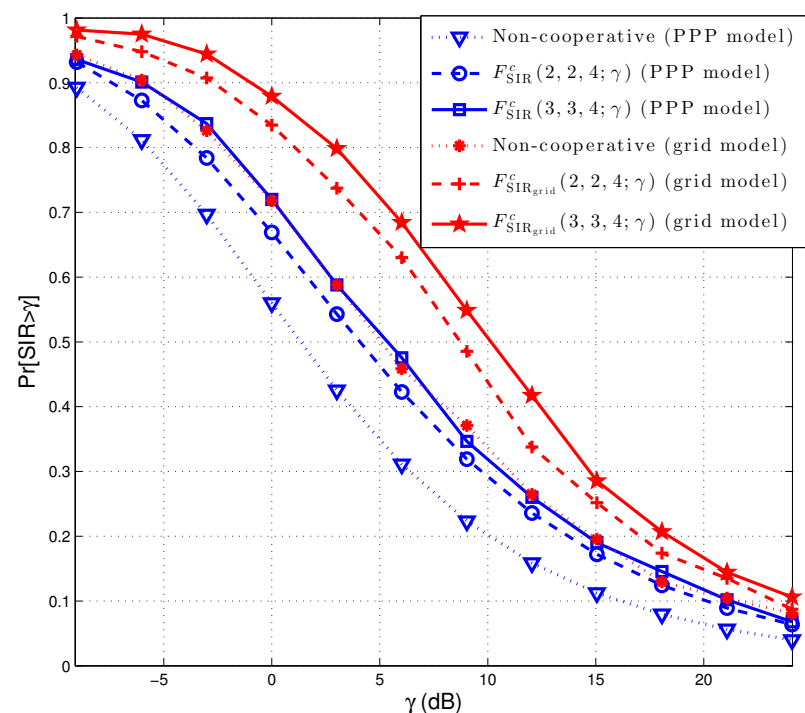

Fig. 8. CCDF of the SIR with coordinated beamforming for both the stochastic and the deterministic grid models, with $\beta=4$.

typical user is small and that it scales with the fading coherence.

\section{APPENDIX A}

\section{PROOF OF THEOREM 1}

The proof relies on two Lemmas, reproduced next for the sake of completeness.

Lemma 2. [Fading distribution] The fading distribution of the desired link of a given user, $H_{1}=\left|\mathbf{h}_{1,1} \mathbf{v}_{1}\right|^{2}$, is chi-squared with $2\left(N_{\mathrm{t}}-K+1\right)$ degrees of freedom while the fading distributions for the out-of-cluster interference links, $H_{k}=\left|\mathbf{h}_{1, k} \mathbf{v}_{k}\right|^{2}$ for $k \in\{K+1, K+2, \ldots\}$, are exponential with unit-mean. Furthermore, all fading terms $H_{k}$ for $k \in\{1, K+1, K+2 \ldots\}$ are mutually independent.

Proof. See [26, Appendix A].

Lemma 3. [PPP distance distribution] Given a PPP in $\mathbb{R}^{2}$ with intensity $\lambda$, the distribution of the distance $\left\|d_{K}\right\|$ between a typical user and its Kth serving $B S$ is the generalized Gamma distribution

$$
f_{\left\|d_{K}\right\|}(r)=\frac{2\left(\lambda \pi r^{2}\right)^{K}}{r \Gamma(K)} e^{-\lambda \pi r^{2}}
$$

where $\Gamma(K)=\int_{0}^{\infty} e^{-x} x^{K-1} \mathrm{~d} x$ is the Gamma function. Proof. See [24].

Let us denote by

$$
I_{r}=\sum_{d_{k} \in \Phi / \mathcal{B}(0, r)} H_{k}\left\|d_{k}\right\|^{-\beta}
$$

the aggregate interference power from all the out-ofcluster BSs, conditioned on the $K$ th in-cluster BS location satisfying $\left\|d_{K}\right\|=r$ where $\mathcal{B}(0, r)$ denotes a circle centered at the origin with radius $r$. Then, the conditional CCDF of the SIR is given by

$$
\begin{aligned}
& F_{\mathrm{SIR} \mid \delta_{1}}^{\mathrm{c}}\left(K, N_{\mathrm{t}}, \beta, \delta_{1} ; \gamma\right) \\
& =\mathbb{E}\left[\mathbb{P}\left[\frac{\left(H_{1} \delta_{1}^{-\beta}\right) r^{-\beta}}{I_{r}} \geq \gamma \mid \delta_{1},\left\|d_{K}\right\|=r\right] \mid \delta_{1}\right] \\
& =\mathbb{E}\left[\mathbb{P}\left[H_{1} \geq \delta_{1}^{\beta} r^{\beta} \gamma I_{r} \mid \delta_{1},\left\|d_{K}\right\|=r\right] \mid \delta_{1}\right] \\
& =\mathbb{E}\left[\mathbb{P}\left[H_{1} \geq r^{\beta} \tilde{I}_{r} \mid \delta_{1},\left\|d_{K}\right\|=r\right] \mid \delta_{1}\right]
\end{aligned}
$$

where $\tilde{I}_{r}=\delta_{1}^{\beta} \gamma I_{r}$ and the expectation is over the distribution of $r$. From Lemma 2, since $H_{1}=\left|\mathbf{h}_{1,1} \mathbf{v}_{1}\right|^{2}$ is chi-squared with $N_{\mathrm{t}}-K+1$ degrees of freedom, we obtain

$$
\begin{aligned}
& F_{\mathrm{SIR}}^{\mathrm{c}}\left(K, N_{\mathrm{t}}, \beta, \delta_{1} ; \gamma\right) \\
& =\mathbb{E}\left[\mathbb{E}\left[\sum_{m=0}^{N_{\mathrm{t}}-K} \frac{r^{\beta m}}{m !} \tilde{I}_{r}^{m} e^{-r^{\beta} \tilde{I}_{r}} \mid \delta_{1},\left\|d_{K}\right\|=r\right] \mid \delta_{1}\right]
\end{aligned}
$$

where the inner expectation is over the distribution of $\tilde{I}_{r}$. From the derivative property of the Laplace transform, which is $\mathbb{E}\left[X^{m} e^{-s X}\right]=(-1)^{m} \frac{\mathrm{d}^{m} \mathcal{L}_{X}(s)}{\mathrm{d} s^{m}}$, we finally obtain

$$
\begin{aligned}
& F_{\mathrm{SIR}}^{\mathrm{c}}\left(K, N_{\mathrm{t}}, \beta, \delta_{1} ; \gamma\right) \\
& =\mathbb{E}\left[\left.\sum_{m=0}^{N_{\mathrm{t}}-K} \frac{r^{\beta m}}{m !}(-1)^{m} \frac{\mathrm{d}^{m} \mathcal{L}_{\tilde{I}_{r}}(s)}{\mathrm{d} s^{m}}\right|_{s=r^{\beta}}\right]
\end{aligned}
$$

which completes the proof.

\section{APPENDIX B}

\section{PROOF OF THEOREM 2}

To prove this result, the following lemma is needed.

Lemma 4. [Alzer's Inequalty [27], [28]] If $H_{1}$ is chisquared with $2 M$ degrees of freedom, then the $C D F$ $F_{H_{1}}(\gamma)=\mathbb{P}\left[H_{1}<\gamma\right]$ is upper and lower bounded by

$$
\left(1-e^{-\kappa \gamma}\right)^{M} \leq F_{H_{1}}(\gamma) \leq\left(1-e^{-\gamma}\right)^{M}
$$

where $F_{H_{1}}(\gamma)=\int_{0}^{\gamma} \frac{e^{-x} x^{M-1}}{(M-1) !} \mathrm{d} x$ and $\kappa=(M !)^{-\frac{1}{M}}$. Strict equalities hold when $M=1$, i.e., when $H_{1}$ is an exponential random variable with mean one.

Now we are ready to prove Theorem 2. We focus on proving the upper bound therein because the lower bound is directly obtained from the former by setting $\kappa=1$. 
Conditioned on the $K$ th BS being located at distance $r$ from the user, the conditional CCDF of the SIR can be written as

$$
\begin{aligned}
& \mathbb{P}\left[\frac{\left(H_{1} \delta_{1}^{-\beta}\right) r^{-\beta}}{I_{r}} \geq \gamma \mid \delta_{1},\left\|d_{K}\right\|=r\right] \\
& =\mathbb{P}\left[H_{1} \geq \delta_{1}^{\beta} r^{\beta} \gamma I_{r} \mid \delta_{1},\left\|d_{K}\right\|=r\right] .
\end{aligned}
$$

Applying Lemma 4 and the binomial expansion,

$$
\begin{aligned}
\mathbb{P}\left[H_{1}>x\right] \leq & 1-\left(1-e^{-\kappa x}\right)^{N_{\mathrm{t}}-K+1} \\
& =\sum_{\ell=1}^{N_{\mathrm{t}}-K+1}\left(\begin{array}{c}
N_{\mathrm{t}}-K+1 \\
\ell
\end{array}\right)(-1)^{\ell+1} e^{-\kappa x \ell}
\end{aligned}
$$

from which the conditional CCDF of the SIR in (45) is upper bounded as

$$
\begin{aligned}
& \mathbb{P}\left[H_{1} \geq \delta_{1}^{\beta} r^{\beta} \gamma I_{r} \mid \delta_{1},\left\|d_{K}\right\|=r\right] \\
& \leq \sum_{\ell=1}^{N_{\mathrm{t}}-K+1}\left(\begin{array}{c}
N_{\mathrm{t}}-K+1 \\
\ell
\end{array}\right)(-1)^{\ell+1} \mathbb{E}\left[e^{-\kappa \ell \delta_{1}^{\beta} r^{\beta} \gamma I_{r}} \mid \delta_{1},\left\|d_{K}\right\|=r\right]
\end{aligned}
$$

where the expectation is over the distribution of $I_{r}$.

Letting $N_{\mathrm{t}}-K+1=M$ and unconditioning with respect to the location of the $K$ th $\mathrm{BS}$,

$$
\begin{aligned}
& F_{\mathrm{SIR}}^{\mathrm{c}}\left(K, N_{\mathrm{t}}, \beta, \delta_{1} ; \gamma\right) \\
& =\mathbb{E}\left[\mathbb{P}\left[\operatorname{SIR}\left(K, N_{\mathrm{t}}, \beta, \delta_{1}\right)>\gamma \mid \delta_{1},\left\|d_{K}\right\|=r\right] \mid \delta_{1}\right] \\
& \leq \sum_{\ell=1}^{M}\left(\begin{array}{c}
M \\
\ell
\end{array}\right)(-1)^{\ell+1} \mathbb{E}\left[\mathbb{E}\left[e^{-\kappa \ell \delta_{1}^{\beta} r^{\beta} \gamma I_{r}} \mid \delta_{1}\left\|d_{K}\right\|=r\right] \mid \delta_{1}\right]
\end{aligned}
$$

with inner and outer expectations over the distributions of $I_{r}$ and $r$, respectively. To evaluate these expectations, we first compute the conditional Laplace transform of $I_{r}$. Conditioned on $\left\|d_{K}\right\|=r$, such Laplace transform is

$$
\begin{aligned}
\mathcal{L}_{I_{r}}(s) & =\mathbb{E}\left[e^{-s I_{r}} \mid\left\|d_{K}\right\|=r\right] \\
& =\mathbb{E}\left[e^{-s \sum_{d_{k} \in \Phi / \mathcal{B}(0, r)} H_{k}\left\|d_{k}\right\|^{-\beta}} \mid\left\|d_{K}\right\|=r\right] \\
& =\mathbb{E}\left[\prod_{d_{k} \in \Phi / \mathcal{B}(0, r)} e^{-s H_{k}\left\|d_{k}\right\|^{-\beta}} \mid\left\|d_{K}\right\|=r\right] \\
& =\mathbb{E}\left[\prod_{d_{k} \in \Phi / \mathcal{B}(0, r)} \frac{1}{1+s\left\|d_{k}\right\|^{-\beta}} \mid\left\|d_{K}\right\|=r\right] \\
& =\exp \left(-2 \pi \lambda \int_{r}^{\infty} \frac{u}{1+s^{-1} u^{\beta}} \mathrm{d} u\right)
\end{aligned}
$$

where (51) follows from the independence of $d_{k}$ and $H_{k}$, (52) holds because $H_{k}$ is exponentially distributed and unit mean for $k \in\{K+1, K+2, \ldots\}$, and (53) follows from the probability generating functional of the PPP. Evaluating this conditional Laplace transform at $s=\kappa \ell \delta_{1}^{\beta} \gamma r^{\beta}$,

$$
\begin{aligned}
& \mathcal{L}_{I_{r}}\left(\kappa \ell \delta_{1}^{\beta} \gamma r^{\beta}\right) \\
& =\exp \left(-2 \pi \lambda \int_{r}^{\infty} \frac{u}{1+\left(\kappa \ell \delta_{1}^{\beta} \gamma\right)^{-1}\left(\frac{u}{r}\right)^{\beta}} \mathrm{d} u\right) \\
& =\exp \left(-\pi \lambda r^{2}\left(\kappa \ell \delta_{1}^{\beta} \gamma\right)^{2 / \beta} \int_{\left(\kappa \ell \delta_{1} \gamma\right)^{\frac{-2}{\beta}}}^{\infty} \frac{1}{1+v^{\beta / 2}} \mathrm{~d} v\right) \\
& =\exp \left(-\pi \lambda r^{2} \mathcal{D}\left(\kappa \ell \delta_{1}^{\beta} \gamma, \beta\right)\right)
\end{aligned}
$$

where (54) follows from the variable change

$$
v=\left[\left(\frac{1}{\kappa \ell \delta_{1} \gamma^{1 / \beta}}\right) \frac{\mu}{r}\right]^{2}
$$

while (55) holds because

$$
\begin{aligned}
\mathcal{D}\left(\kappa \ell \delta_{1}^{\beta} \gamma, \beta\right) & =\left(\kappa \ell \delta_{1}^{\beta} \gamma\right)^{2 / \beta} \int_{\left(\kappa \ell \delta_{1}^{\beta} \gamma\right)^{-2 / \beta}}^{\infty} \frac{1}{1+v^{\beta / 2}} \mathrm{~d} v \\
& =\frac{2 \kappa \ell \delta_{1}^{\beta} \gamma}{\beta-2}{ }_{2} F_{1}\left(1, \frac{\beta-2}{\beta}, 2-\frac{2}{\beta},-\kappa \ell \delta_{1}^{\beta} \gamma\right) .
\end{aligned}
$$

To uncondition the foregoing Laplace transform, we marginalize it with respect to $r$ using the distribution in Lemma 3. With that, the Laplace transform of the aggregate out-of-cluster interference power emerges as

$$
\begin{aligned}
& \mathbb{E}\left[\mathcal{L}_{I}\left(\kappa \ell \delta_{1}^{\beta} \gamma r^{\beta}\right)\right] \\
& =\int_{r>0} \exp \left(-\pi \lambda r^{2} \mathcal{D}\left(\kappa \ell \delta_{1}^{\beta} \gamma, \beta\right)\right) \frac{2\left(\lambda \pi r^{2}\right)^{K}}{r \Gamma(K)} e^{-\lambda \pi r^{2}} \mathrm{~d} r \\
& =\int_{0}^{\infty} e^{-x}\left(\frac{x}{\pi \lambda\left[1+\mathcal{D}\left(\kappa \ell \delta_{1}^{\beta} \gamma, \beta\right)\right]}\right)^{K-1} \\
& =\left[\frac{2(\pi \lambda)^{K}}{\Gamma(K)\left[1+\mathcal{D}\left(\kappa \ell \delta_{1}^{\beta} \gamma, \beta\right)\right] 2 \pi \lambda} \mathrm{d} x\right. \\
& =\left[\frac{1}{1+\mathcal{D}\left(\kappa \ell \delta_{1}^{\beta} \gamma, \beta\right)}\right]^{K}
\end{aligned}
$$

where (58) follows from the variable change

$$
x=\pi \lambda\left[1+\mathcal{D}\left(\kappa \ell \delta_{1}^{\beta} \gamma, \beta\right)\right] r^{2}
$$

whereas (59) follows from the definition of the Gamma function. By plugging (59) into (48), we finally obtain

$$
F_{\text {SIR }}^{\mathrm{c}}\left(K, N_{\mathrm{t}}, \beta, \delta_{1} ; \gamma\right) \leq \sum_{\ell=1}^{N_{\mathrm{t}}-K+1} \frac{\left(\begin{array}{c}
N_{\mathrm{t}}-K+1 \\
\ell
\end{array}\right)(-1)^{\ell+1}}{\left[1+\mathcal{D}\left(\ell \kappa \delta_{1}^{\beta} \gamma, \beta\right)\right]^{K}}
$$

and, by setting $\kappa=1$, we further have the lower bound in (13), which completes the proof. 


\section{APPENDIX C}

ProOF OF LEMMA 1

We start by computing the joint PDF of $\left\|d_{1}\right\|$ and $\left\|d_{K}\right\|$. Consider the four nonoverlapping areas $A_{1}=\mathcal{B}\left(0, r_{1}\right), A_{2}=\mathcal{B}\left(0, r_{1}+\mathrm{d} r_{1}\right) / \mathcal{B}\left(0, r_{1}\right)$, $A_{3}=\mathcal{B}\left(0, r_{K}\right) / \mathcal{B}\left(0, r_{1}+\mathrm{d} r_{1}\right)$, and $A_{4}=\mathcal{B}\left(0, r_{K}+\right.$ $\left.\mathrm{d} r_{K}\right) / \mathcal{B}\left(0, r_{K}\right)$. By definition of the PPP, the joint probability that $\left\|d_{1}\right\|$ and $\left\|d_{K}\right\|$ belong to the two (thin ring) areas $A_{2}$ and $A_{4}$, respectively, is given by the product of the four independent probability events as

$\mathbb{P}\left[\left\|d_{1}\right\| \in A_{2},\left\|d_{K}\right\| \in A_{4}\right]= \begin{cases}P_{1} P_{2} P_{3} P_{4} & \text { if } r_{1} \leq r_{K} \\ 0 & \text { otherwise }\end{cases}$

where

$P_{1}=\mathbb{P}\left[\right.$ No points in $\left.A_{1}\right]=e^{-\lambda \pi r_{1}^{2}}$

$P_{2}=\mathbb{P}\left[\right.$ One point in $\left.A_{2}\right]=\lambda \pi 2 r_{1} \mathrm{~d} r_{1} e^{-\lambda \pi 2 r_{1} \mathrm{~d} r_{1}}$

$P_{3}=\mathbb{P}\left[K-2\right.$ points in $\left.A_{3}\right]$

$$
=\frac{(\lambda \pi)^{K-2}}{(K-2) !}\left[r_{K}^{2}-\left(r_{1}+\mathrm{d} r_{1}\right)^{2}\right]^{K-2} e^{-\lambda \pi 2\left[r_{K}^{2}-\left(r_{1}+\mathrm{d} r_{1}\right)^{2}\right]}
$$

$P_{4}=\mathbb{P}\left[\right.$ One point in $\left.A_{4}\right]=\lambda \pi 2 r_{K} \mathrm{~d} r_{K} e^{-\lambda \pi 2 r_{K} \mathrm{~d} r_{K}}$.

From the limits of the joint probability in (62), the joint PDF of $\left\|d_{1}\right\|$ and $\left\|d_{K}\right\|$ emerges as

$$
\begin{gathered}
f_{\left\|d_{1}\right\|,\left\|d_{K}\right\|}\left(r_{1}, r_{K}\right)=\lim _{\mathrm{d} r_{1}, \mathrm{~d} r_{K} \rightarrow 0} \frac{\mathbb{P}\left[\left\|d_{1}\right\| \in A_{2},\left\|d_{K}\right\| \in A_{4}\right]}{\mathrm{d} r_{1} \mathrm{~d} r_{K}} \\
= \begin{cases}\frac{4(\lambda \pi)^{K}}{(K-2) !} r_{1} r_{K}\left(r_{K}^{2}-r_{1}^{2}\right)^{K-2} e^{-\lambda \pi r_{K}^{2}} & \text { if } r_{1} \leq r_{K} \\
0 & \text { otherwise }\end{cases}
\end{gathered}
$$

Utilizing the joint PDF of $\left\|d_{1}\right\|$ and $\left\|d_{K}\right\|$, we derive the CDF of $\delta_{1}=\frac{\left\|d_{1}\right\|}{\left\|d_{K}\right\|}$ as

$$
\begin{aligned}
\mathbb{P}\left[\delta_{1} \leq x\right] & =\mathbb{P}\left[\frac{\left\|d_{1}\right\|}{\left\|d_{K}\right\|} \leq x\right] \\
& =\mathbb{P}\left[\left\|d_{1}\right\| \leq x\left\|d_{K}\right\|\right] \\
& =\int_{0}^{\infty} \int_{0}^{x r_{K}} f_{\left\|d_{1}\right\|,\left\|d_{K}\right\|}\left(r_{1}, r_{K}\right) \mathrm{d} r_{1} \mathrm{~d} r_{K}
\end{aligned}
$$

$=\int_{0}^{\infty} \int_{0}^{x r_{K}} \frac{4(\lambda \pi)^{K}}{(K-2) !} r_{1} r_{K}\left(r_{K}^{2}-r_{1}^{2}\right)^{K-2} e^{-\lambda \pi r_{K}^{2}} \mathrm{~d} r_{1} \mathrm{~d} r_{K}$

$$
=1-\left(1-x^{2}\right)^{K-1}
$$

where $0 \leq x \leq 1$. Therefore, the PDF of $\delta_{1}$ is given by

$$
\begin{aligned}
f_{\delta_{1}}(x) & =\frac{\mathrm{d} \mathbb{P}\left[\delta_{1} \leq x\right]}{\mathrm{d} x} \\
& =2(K-1) x\left(1-x^{2}\right)^{K-2} .
\end{aligned}
$$

\section{APPENDIX D}

PROOF OF EQ. (25)

Recall that

$$
\mathcal{D}\left(\tilde{\gamma} \delta_{1}^{\beta}, \beta\right)=\tilde{\gamma}^{2 / \beta} \delta_{1}^{2} \int_{1 /\left(\tilde{\gamma}^{2 / \beta} \delta_{1}^{2}\right)}^{\infty} \frac{1}{1+v^{\frac{\beta}{2}}} \mathrm{~d} v .
$$

where $\delta_{1}$ is distributed as per Lemma 1 . We approximate the integral above as a constant value that captures the effect of the randomness induced by $\delta_{1}$,

$$
\mathbb{E}\left[\int_{1 /\left(\tilde{\gamma}^{2 / \beta} \delta_{1}^{2}\right)}^{\infty} \frac{1}{1+v^{\frac{\beta}{2}}} \mathrm{~d} v\right] \simeq \frac{1}{\sqrt{K}} \mathcal{A}\left(\frac{\sqrt{K}}{\tilde{\gamma}^{2 / \beta}}\right)
$$

where the expectation is over $\delta_{1}$. From (70),

$$
\mathcal{D}\left(\tilde{\gamma} \delta_{1}^{\beta}, \beta\right) \simeq \frac{\tilde{\gamma}^{2 / \beta} \delta_{1}^{2}}{\sqrt{K}} \mathcal{A}\left(\frac{\sqrt{K}}{\tilde{\gamma}^{2 / \beta}}\right) .
$$

Plugging (71) into the left side of (25) and marginalizing with respect to $\delta_{1}$,

$$
\begin{aligned}
& \int_{0}^{1} \frac{2(K-1) x\left(1-x^{2}\right)^{K-2}}{\left[1+\mathcal{D}\left(\tilde{\gamma} x^{\beta}, \beta\right)\right]^{K}} \mathrm{~d} x \\
& \simeq \int_{0}^{1} \frac{2(K-1) x\left(1-x^{2}\right)^{K-2}}{\left[1+\frac{\tilde{\gamma}^{2 / \beta}}{\sqrt{K}} \mathcal{A}\left(\frac{\sqrt{K}}{\tilde{\gamma}^{2 / \beta}}\right) x^{2}\right]^{K}} \mathrm{~d} x \\
& =\frac{1}{1+\frac{\tilde{\gamma}^{2 / \beta}}{\sqrt{K}} \mathcal{A}\left(\frac{\sqrt{K}}{\tilde{\gamma}^{2 / \beta}}\right)} .
\end{aligned}
$$

\section{APPENDIX E}

\section{ProOF OF PROPOSITION 1}

Recall that the upper bound in Thm. 2 is exact for $K=N_{\mathrm{t}}$. The outage probability $P_{\text {out }}\left(K, K, \beta, \delta_{1} ; \gamma\right)=$ $1-F_{\mathrm{SIR}}^{\mathrm{c}}\left(K, K, \beta, \delta_{1} ; \gamma\right)$ expands at $\gamma=0$ as

$$
\begin{aligned}
P_{\text {out }}\left(K, K, \beta, \delta_{1} ; \gamma\right) & =1-F_{\mathrm{SIR}}^{\mathrm{c}}\left(K, K, \beta, \delta_{1} ; \gamma\right) \\
& =1-\frac{1}{\left(1+\frac{\delta_{1}^{\beta} \gamma}{\beta-2}\right)^{K}}+o(\gamma)^{2} \\
& =1-\left(1-\frac{K \delta_{1}^{\beta} \gamma}{\beta-2}\right)+o(\gamma)^{2}
\end{aligned}
$$

where we have invoked the series expansion of the Gauss hypergeometric function at $\gamma=0$,

$$
\mathcal{D}\left(\delta_{1}^{\beta} \gamma, \beta\right)=\frac{\delta_{1}^{\beta} \gamma}{\beta-2}+o(\gamma)^{2}
$$

as well as

$$
\frac{1}{(1+x)^{K}}=1-K x+o(x)^{2} .
$$


By dropping the second order error term and marginalizing with respect to $\delta_{1}$, we obtain the average outage probability

$$
\begin{aligned}
& 1-F_{\mathrm{SIR}}^{\mathrm{c}}(K, K, \beta ; \gamma) \\
& =\frac{K \mathbb{E}\left[\delta_{1}^{\beta}\right] \gamma}{\beta-2+o(\gamma)} \\
& =\frac{K \gamma \int_{0}^{1} \delta_{1}^{\beta} f_{\delta_{1}}(x) \mathrm{d} x}{\beta-2}+o(\gamma) \\
& =\frac{2 K(K-1) \gamma \int_{0}^{1} x^{\beta+1}\left(1-x^{2}\right)^{K-2} \mathrm{~d} x}{\beta-2}+o(\gamma) \\
& =\frac{K(K-1)}{\beta-2} \frac{\Gamma\left(\frac{\beta}{2}+1\right) \Gamma(K-1)}{\Gamma\left(\frac{\beta}{2}+K\right)} \gamma+o(\gamma)
\end{aligned}
$$

which completes the proof.

\section{REFERENCES}

[1] N. Lee, D. Morales, R. W. Heath Jr., and A. Lozano, "Coordinated Beamforming with Dynamic Clustering: A Stochastic Geometry Approach," IEEE Int. Conf. in Communications (ICC'14), June 2014.

[2] "Coordinated multi-point operation for LTE physical layer aspects (Release 11)," 3GPP TR 36.819, 2011.

[3] B. Clerckx, H. Lee, Y-J. Hong, and G. Kim, "A practical cooperative multi-cell MIMO-OFDMA network based on rank coordination," IEEE Trans. Wireless Communications, vol. 12, no. 4 pp. 1481 - 1491, Apr. 2013.

[4] D. Gesbert, S. Hanly, H. Huang, S. Shamai, O. Simeone, and W. Yu, "Multi-cell MIMO cooperative networks: A new look at interference," IEEE Journal on Sel. Areas in Communications, vol. 28, no. 9, pp. 1380-1408, Dec. 2010.

[5] M. K. Karakayali, G. J. Foschini, and R. A. Valenzuela, "Network coordination for spectrally efficient communications in cellular systems," IEEE Trans. Wireless Communications, vol. 13, no. 4, pp. 56 - 61, Aug. 2006.

[6] S. Venkatesan, H. C. Huang, A. Lozano, R. A. Valenzuela, "A WiMAX-based implementation of network MIMO for indoor wireless systems," EURASIP Journal on Advances in Signal Processing, vol. 2009, Feb. 2009.

[7] V. R. Cadambe and S. A. Jafar, "Interference alignment and the degrees of freedom for the $K$ user interference channel," IEEE Trans. Inform. Theory, vol. 54, no. 8, pp. 3425 - 3441, Aug. 2008.

[8] O. Somekh, O. Simeone, Y. Bar-Ness, A. M. Haimovich, and S. Shamai, "Cooperative multi-cell zero-forcing beamforming in cellular downlink channels," IEEE Trans. Inform. Theory, vol. 55, no. 7, pp. 3206 - 3219, Jul. 2009.

[9] H. Huh, A. M. Tulino, and G. Caire, "Network MIMO with linear zero-forcing beamforming: Large system analysis, impact of channel estimation, and reduced-complexity scheduling," IEEE Trans. Inform. Theory, vol. 58, no. 5, pp. 2911 - 2934, May 2012.

[10] A. Lozano, J. G. Andrews, and R. W. Heath, Jr., "Fundamental limits of cooperation," IEEE Trans. Inform. Theory, vol. 59, no. 9, pp. 5213-5226, Sept. 2013.

[11] H. Huang, M. Trivellato, A. Hottinen, M. Sha, P. Smith, and R. Valenzuela, "Increasing downlink cellular throughput with limited network MIMO coordination," IEEE Trans. Wireless Communications, vol. 8, no. 6, pp. 2983 - 2989, June 2009.
[12] J. Zhang, R. Chen, J. G. Andrews, A. Ghosh, and R. W. Heath Jr., "Networked MIMO with clustered linear precoding," IEEE Trans. Wireless Communications, vol. 8, pp. 1910 - 1921, Apr. 2009.

[13] J. Xu, J. Zhang, and J. G. Andrews, "On the accuracy of the Wyner model in downlink cellular networks," IEEE Trans. Wireless Communications, vol. 10, pp. 3098 - 3109, Sep. 2011.

[14] O. Simeone, N. Levy, A. Sanderovich, O. Somekh, B. M. Zaidel, H. V. Poor, and S. Shamai, "Information theoretic considerations for wireless cellular systems: The impact of cooperation," Foundations and Trends in Communications and Information Theory, vol. 7, 2012.

[15] J. G. Andrews, F. Baccelli, and R. K. Ganti, "A tractable approach to coverage and rate in cellular networks," IEEE Trans. on Communications, vol. 59, no. 11, pp. 3122 - 3134, Nov. 2011.

[16] K. Huang and J. G. Andrews, "An analytical framework for multi-cell coordination via stochastic geometry and large deviations," IEEE Trans. Inform. Theory, vol. 59, no. 4, pp. 2501 - 2516, April 2013.

[17] A. Giovanidis and F. Baccelli, "A stochastic geometry framework for analyzing pairwise-cooperative cellular networks," Submitted to IEEE Trans. Inform. Theory [online:http://arxiv.org/abs/1305.6254]

[18] S. Akoum and R. W. Heath, Jr, "Multi-cell coordination: A stochastic geometry approach," IEEE 13th International Workshop on Signal Processing Advances in Wireless Communications (SPAWC), pp. 16-20, June. 2012.

[19] A. Papadogiannis, D. Gesbert, and E. Hardouin, "A dynamic clustering approach in wireless networks with multi-cell cooperative processing," in Proc. IEEE Int'l Conf. on Comm., pp. 4033 - 4037, May 2008.

[20] R. Mungara, G. George, and A. Lozano, "System-level performance of distributed cooperation," in Proc. Asilomar Conf. on Signals, Systems and Computers, pp. 1561 - 1565, Nov. 2012.

[21] R. Mungara, G. George, and A. Lozano, "System-level performance of interference alignment," IEEE Trans. on Wireless Communications, to appear.

[22] S. Kaviani and W. A. Krzymien, "Multi-cell scheduling in network MIMO," In Proc. IEEE Global Telecomm. Conf. (GLOBECOM10), pp. 1 - 5, Dec. 2010.

[23] N. Lee, R. W. Heath Jr., D. Morales, and A. Lozano, "Base station cooperation with dynamic clustering in super-dense cloudRAN," IEEE GLOBECOM Workshop, Dec. 2013.

[24] M. Haenggi, "On distances in uniformly random networks," IEEE Trans. Inform. Theory, vol. 51, no. 10, pp. 3584 - 3586, Oct. 2005.

[25] S. Nadarajah, "A review of results on sums of random variables," ACTA Appl. Math., vol. 103, no. 2, pp. 131 - 140, 2008.

[26] N. Jindal, J. G. Andrews, and S. Weber, "Multi-antenna communication in ad hoc networks: achieving MIMO gains with SIMO transmission," IEEE Transactions on Communications, vol. 59, pp. 529 - 540, February 2011.

[27] K. Huang, J. G. Andrews, and R. W. Heath, Jr., "Space division multiple access with a sum feedback rate constraint," IEEE Transactions on Communications, vol. 55, no. 7, pp. 3879 - 3891, July 2007.

[28] H. Alzer, "On some inequalities for the incomplete Gamma function," Math. Comput., vol. 66, no. 218, pp. 771 - 778, 2005.

[29] N. Jindal and A. Lozano, "A unified treatment of optimum pilot overhead in multipath fading channels," IEEE Trans. Communications, vol. 58, no. 10, pp. 2939-2948, Oct. 2010.

[30] A. Lozano and N. Jindal, "Are yesterday's information-theoretic fading models and performance metrics adequate for the analysis of today's wireless systems?," IEEE Communications Magazine, vol. 50, no. 11, pp. 210-217, Nov. 2012. 